\title{
Stress-induced Reversal of MicroRNA Repression and mRNA P-body Localization in Human Cells
}

\author{
S.N. Bhattacharyya, ${ }^{*}$ R. Habermacher, ${ }^{*}$ U. Martine $^{\dagger}$ E.I. Closs,${ }^{\dagger}$ and W. Filipowicz* \\ ${ }^{*}$ Friedrich Miescher Institute for Biomedical Research, 4002 Basel, Switzerland; ${ }^{\dagger}$ Department of Pharmacology, \\ Johannes Gutenberg University, 67, 55101 Mainz, Germany
}

\begin{abstract}
In metazoa, microRNAs (miRNAs) imperfectly base-pair with the $3^{\prime}$-untranslated region (3'UTR) of mRNAs and prevent protein accumulation by either repressing translation or inducing mRNA degradation. Examples of specific mRNAs undergoing miRNA-mediated repression are numerous, but whether the repression is a reversible process remains largely unknown. Here, we show that cationic amino acid transporter 1 (CAT-1) mRNA and reporters bearing the CAT-1 3'UTR or its fragments can be relieved from the miRNA miR-122-induced inhibition in human hepatoma cells in response to different stress conditions. The derepression of CAT-1 mRNA is accompanied by its release from cytoplasmic processing bodies (P bodies) and its recruitment to polysomes, indicating that $\mathrm{P}$ bodies act as storage sites for mRNAs inhibited by miRNAs. The derepression requires binding of HuR, an AU-rich-element-binding ELAV family protein, to the 3'UTR of CAT-1 mRNA. We propose that proteins interacting with the 3'UTR will generally act as modifiers altering the potential of miRNAs to repress gene expression.
\end{abstract}

miRNAs are 20- to 22-nucleotide-long regulatory RNAs expressed in plants and metazoan animals. Current estimates indicate that hundreds of different miRNAs are encoded in individual genomes, with the number of human miRNAs possibly reaching 1000 . Approximately $30 \%$ of all human genes are predicted to be subject to miRNA regulation. Specific functions and target mRNAs have been assigned to only a few dozen miRNAs, but it is apparent that miRNAs participate in the regulation of many different biological processes. Changes in miRNA expression are observed in human pathologies and some miRNAs were shown to act as oncogenes or tumor supressors (for review, see Ambros 2004; Bartel 2004; Wienholds and Plasterk 2005).

miRNAs regulate gene expression posttranscriptionally, by base-pairing to target mRNAs. In animals, most investigated miRNAs form imperfect hybrids with sequences in the $3^{\prime}$ UTR, with the miRNA 5'-proximal "seed" region (positions $2-8$ ) providing most of the pairing specificity (for review, see Filipowicz 2005; Tomari and Zamore 2005). The miRNA association results in translational repression, frequently accompanied by a considerable degradation of the mRNA by a non-RNAi mechanism (for review, see Pillai 2005; Valencia-Sanchez et al. 2006).

Argonaute (AGO) proteins are the essential and bestcharacterized components of miRNPs. In mammals, only one of the four AGO proteins, AGO2, is competent to catalyze cleavage of mRNA in the RNA interference (RNAi)like mechanism (Liu et al. 2004; Meister et al. 2004). On the other hand, all four AGO proteins, AGO1-4, appear to function in miRNA repression (Liu et al. 2004; Meister et al. 2004; Pillai et al. 2004). The mechanism of translational inhibition by miRNAs is not well understood. Some natural or model miRNAs interfere with the initiation of protein synthesis (Humphreys et al. 2005; Pillai et al. 2005), although others may affect more downstream steps in translation (Olsen and Ambros 1999; Petersen et al. 2006). The
AGO proteins and repressed mRNAs are enriched in the cytoplasmic P bodies (Jakymiw et al. 2005; Liu et al. 2005; Pillai et al. 2005). The P-body association may represent a secondary event, which follows the translation inhibition step. Since mRNA catabolic enzymes also reside in P bodies, the relocation likely results in the reported mRNA degradation (Pillai 2005; Valencia-Sanchez et al. 2006).

To date, miRNAs have been primarily identified as negative regulators of expression of cellular mRNAs, and it remains unknown whether the inhibition of a specific mRNA can be effectively reversed. Clearly, the ability to disengage miRNPs from the repressed mRNA, or render them inactive, would make miRNA regulation much more dynamic and also more responsive to specific cellular needs. In this work, we present evidence that CAT-1 mRNA, which encodes the high-affinity cationic amino acid transporter and which is translationally repressed by miR-122 in Huh7 hepatoma cells, can be relieved from the miR-122 repression by subjecting Huh7 cells to different stress conditions. The derepression is accompanied by the release of CAT-1 mRNA from P bodies and its recruitment to polysomes, consistent with miR-122 inhibiting translational initiation. We provide evidence that the stress-induced up-regulation is mediated by binding of $\mathrm{HuR}$, an AU-rich element (ARE)-binding protein, to the $3^{\prime}$ UTR of CAT-1 mRNA.

\section{RESULTS}

\section{CAT-1 mRNA as a Model to Investigate Reversibility of miRNA-mediated Repression}

To investigate whether mRNA can be relieved from miRNA-mediated repression, we looked at the mRNA encoding the high-affinity cationic amino acid transporter, CAT-1, a member of the CAT (or SLC7A1-4) family of system $\mathrm{y}^{+}$transporters. CAT-1, which facili- 
tates uptake of arginine and lysine in mammalian cells, is expressed ubiquitously, but its levels vary significantly in different cells and tissues and its expression is known to undergo extensive regulation at both transcriptional and posttranscriptional levels (for review, see Hatzoglou et al. 2004). CAT-1 regulation has been studied intensively in rat C6 glioma cells, where transcription of the gene and stability and translation of the mRNA are up-regulated in response to different types of cellular stress (Yaman et al. 2002; Haztoglou et al. 2004). In human hepatoma Huh7 cells, CAT- 1 mRNA activity is regulated by a liver-specific miRNA, miR-122. The human CAT-1 3'UTR contains several potential target sites for miR-122 (see Fig. 2A). Experiments performed with endogenous CAT-1 mRNA (Bhattacharyya et al. 2006) and reporters containing its 3 'UTR (or fragments thereof) indicated that miR122 has a repressive effect on translation and, in the case in some chimeric reporter mRNAs, may also cause limited destabilization of the target RNA (Chang et al. 2004; Bhattacharyya et al. 2006). Maintenance of low CAT-1 activity in liver cells is important to avoid hydrolysis of the plasma arginine by arginase, a highly expressed enzyme in hepatocytes, which catalyzes the last step of the urea cycle (hydrolysis of arginine to ornithine and urea). However, under certain conditions, e.g., when urea cycle enzymes are down-regulated or during liver regeneration, CAT-1 expression is induced, most likely to sustain hepatocellular protein synthesis.

\section{Amino Acid Deprivation Induces Translational Up-regulation of CAT-1 mRNA in Huh7 Cells}

In cultured rat C6 glioma cells, CAT-1 protein expression increases in response to amino acid deprivation and other forms of cellular stress. Both transcriptional and posttranscriptional regulation were reported to contribute to the increase (Hatzoglou et al. 2004). We investigated the effect of amino acid starvation on expression of CAT1 protein and mRNA in hepatoma Huh7 cells. The CAT1 protein level increased markedly after 1 hour of starvation and then remained unchanged during several additional hours of amino acid depletion (Fig. 1A). In con-
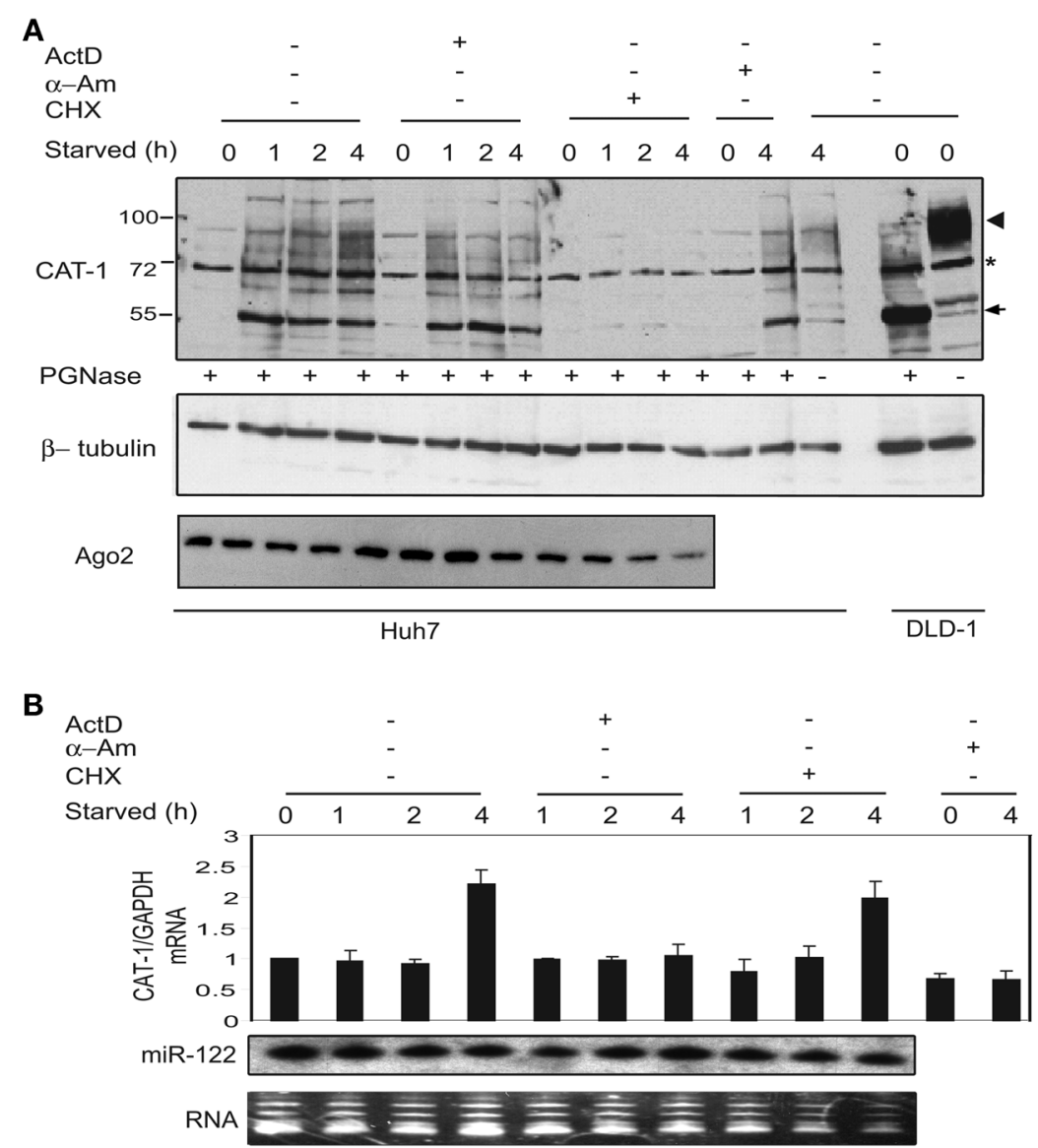

Figure 1. Starvation of Huh7 cells induces expression of CAT-1 protein independent of transcription. $(A)$ Stress-induced expression of CAT-1 in Huh7 cells is a posttranscriptional event. Huh7 cells were cultured for $0-4$ hours in amino-acid-depleted medium in the absence or presence of indicated inhibitors. Proteins were treated with PGNase F and analyzed by western blotting using indicated antibodies. Two lanes on the right contained protein extracts of DLD-1 cells, abundant in CAT-1. Positions of glycosylated and deglycosylated CAT-1 are indicated by an arrowhead and arrow. $(B)$ Analysis of RNA isolated from Huh7 cells starved of amino acids in the presence of indicated inhibitors. (Upper panel) Real-time PCR quantification of CAT-1 mRNA. Normalized values are means (+/-SD) from three independent experiments, with GAPDH mRNA serving as an internal control. (Two lower panels) Northern analysis of miR-122 and staining of the gel with ethidium bromide. Details of methodological procedures used for experiments described in this and other figures are described in Bhattacharyya et al. (2006). (Reprinted, with permission, from Bhattacharyya et al. 2006 [C Elsevier].) 
trast, the substantial increase in CAT-1 mRNA level, measured by either real-time polymerase chain reaction (PCR) (Fig. 1B) or northern blotting (not shown) (Bhattacharyya et al. 2006), was only detectable after 3-4 hours of starvation. In HepG2 hepatoma cells, which do not express miRNA miR-122, no appreciable change in either CAT-1 protein or mRNA level was observed during 4 hours of starvation (data not shown). The early induction of the CAT-1 protein in Huh7 cells was independent of RNA polymerase II transcription, since treatment with inhibitors of RNA polymerase II, either actinomycin D (ActD) or $\alpha$-amanitin ( $\alpha$-Am), had no effect (Fig. 1A). However, the protein accumulation was inhibited by cycloheximide (CHX), an inhibitor of translational elongation (Fig. 1A). Amino acid starvation and treatment of cells with ActD or $\alpha$-Am had no effect on the level of miR-122 or Ago2, essential components of miRNPs (Fig. 1A,B). However, inclusion of either inhibitor prevented the late (4-hour time point) accumulation of CAT-1 mRNA, demonstrating that they effectively inhibit RNA polymerase II transcription in Huh7 cells.

It was important to exclude the possibility that CAT-1 protein turns over rapidly in cells grown in the medium rich in amino acids, and its apparent induction by starvation is due to an increase in protein stability. To this end, we have performed western analysis of lysates prepared from cells grown in either the presence or absence of $\mathrm{CXH}$. The analysis indicated that instead of stabilizing the CAT-1 protein, the starvation increased its turnover (Bhattacharyya et al. 2006). The accelerated decay of CAT-1 in stressed cells provides a plausible explanation for the observation that steady-state levels of the protein do not continue to increase at times beyond 1 hour poststarvation. Taken together, the data indicate that starvation of Huh7 cells results in a de novo synthesis of the CAT-1 protein from the preexisting mRNA pool.

\section{Response to Amino Acid Starvation and Other Types of Stress Is Mediated by the CAT-1 3'UTR and Involves miR-122}

To test whether the translational induction described above is mediated by the CAT- 1 mRNA 3'UTR, we measured the effect of starvation on the activity of different RL-cat reporters (Fig. 2A) in Huh7 cells. In RLcatA, the RL-coding region is fused to the $2.5-\mathrm{kb} 3^{\prime} \mathrm{UTR}$ found in a short form of CAT-1 mRNA. In RL-catB, the 3 '-proximal $1-\mathrm{kb}$ region (referred to as region $\mathrm{D}$ ) of the CAT-1 3'UTR is deleted. The remaining part contains three predicted miR-122-binding sites identified in the

A
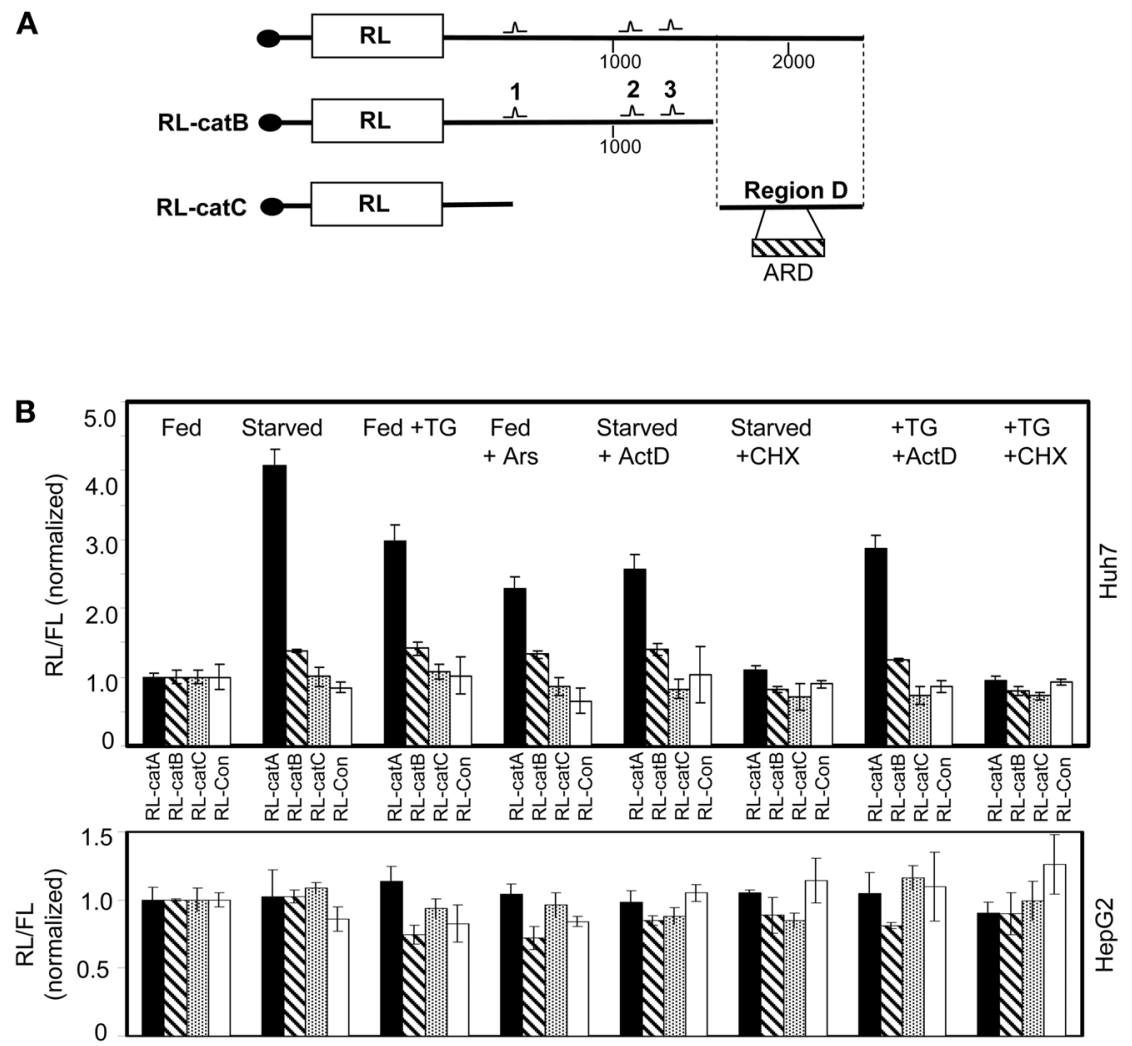

Figure 2. Effect of different types of cellular stress on activity of RL reporters in Huh7 and HepG2 cells. $(A)$ Schemes of reporters bearing different segments of the CAT-1 3'UTR fused to the RL-coding region. Positions of potential miR-122-binding sites (1-3) and the approximately 1-kb region D and its AU-rich subregion ARD are indicated. $(B)$ Expression of the RL-catA reporter is specifically up-regulated in stressed Huh7 cells (upper panel) but not HepG2 cells (lower panel). Cells transfected with indicated reporters were starved for 2 hours (Starved) or grown in the presence of either thapsigargin (TG; 2 hr) or arsenite (Ars; 30 min). ActD or CHX was added at the time of the shift to stress conditions. Values are normalized to activities in nonstressed (Fed) cells, which were set to 1. (Reprinted, with permission, from Bhattacharyya et al. 2006 [C Elsevier].) 
2.5-kb CAT-1 3'UTR. In RL-catC, the CAT-1 3'UTR is shortened further to eliminate the region containing the miR-122 sites. Reporter activity, normalized to activity of the coexpressed firefly luciferase (FL), was tested in transfected Huh7 and HepG2 cells (Fig. 2B). In Huh7 cells, an approximately fourfold induction of RL activity was observed upon starvation of cells transfected with the reporter containing miR-122 sites, RL-catA, but not with that devoid of miRNA sites, RL-catC. Starvation increased expression of RL-catB by approximately $30 \%$ (Fig. 2B). As in the case of endogenous CAT-1 protein, the stress-induced expression of RL from RL-catA was inhibited by addition of CHX but not ActD (Fig. 2B). Likewise, other stress conditions such as the ER stress (induced by thapsigargin) or oxidative stress (induced by arsenite) stimulated expression of RL-catA approximately 2.5 -fold, whereas the effect on other reporters was either minimal or absent (Fig. 2B). Amino acid starvation and treatment with thapsigargin or arsenite had no effect on the level of RL-catA mRNA, indicating that the effect was posttranscriptional (Bhattacharyya et al. 2006).

To test whether the stress-mediated activation of RLcatA indeed involves miR-122, we investigated whether responses observed in Huh7 cells can be reproduced in HepG2 cells, which do not express miR-122. Exposure of HepG2 cells to different forms of stress had no effect on expression of any RL-cat reporter (Fig. 2B, lower panel). However, when HepG2 cells were cotransfected with miR-122, up-regulation of RL-catA was clearly evident in starved cells, with activity of RL-catB and RL-catC remaining unchanged (Bhattacharyya et al. 2006).

\section{HuR Protein Interacts with CAT-1 3'UTR and Is Essential for the Derepression}

The stress-inducible RL-catA reporter differs from the reporter not undergoing activation, RL-catB, by the presence of an additional segment (region $\mathrm{D}$ ) of the CAT-1 3'UTR (Fig. 2A). We found that region D is essential for the stress-induced relief of the miR-122mediated repression in Huh7 cells. Moreover, this region can confer stress inducibility on a reporter inhibited by let-7 RNA, an abundant miRNA expressed in HeLa cells. The let-7-specific RL-3xBulge reporter (Pillai et al. 2005) was used to further dissect CAT-1 region $\mathrm{D}$. It was found that the central part of region $\mathrm{D}$, containing sequences rich in $\mathrm{A}+\mathrm{U}$ and $\mathrm{U}$ residues and referred to hereafter as region ARD (see Fig. 2A), is essential for mediating the stress-induced reversal of miRNA repression (Bhattacharyya et al. 2006).

$\mathrm{HuR}$ is an AU-rich element (ARE)-binding protein, a member of the ELAV family of proteins, implicated in different aspects of posttranscriptional regulation (for review, see Brennan and Steitz 2001; Katsanou et al. 2005; Lal et al. 2005). In response to various types of cellular stress, HuR is mobilized from the nucleus to the cytosol, where it may modulate translation or increase the stability of different target mRNAs, including CAT1 mRNA in rat glioma cells (Yaman et al. 2002). We found that HuR relocates from the nucleus to the cytosol upon amino acid starvation also in Huh7 cells (Fig. 3A) and that the RNA-mediated depletion of HuR, using two different small interfering RNAs (siRNAs) (Fig. 3B), eliminates the RL-catA response in comparison to control cells or cells treated with control siRNA (Fig. 3C). We tested, by native gel analysis, if the ${ }^{32} \mathrm{P}$-labeled ARD fragment, which is essential for mediating the stressinduced derepression, can interact with a recombinant GST-HuR fusion protein. As shown in Figure 3D, purified GST-HuR but not GST, formed a complex with the ARD fragment, and the complex was competed by an excess of unlabeled fragment ARD but not the $\triangle A R D$ portion of region D. Additional experiments indicated that CAT-1 mRNA can be specifically immunoselected from the soluble cytoplasmic fraction of starved Huh7 cells by the anti-HuR antibody. We have also demonstrated that HuR binding per se does not cause activation of the RL reporter devoid of miR-122 sites and not repressed by the miRNA (Bhattacharyya et al. 2006).

Taken together, the above experiments indicate that $\mathrm{HuR}$ has a role in the stress-induced activation of CAT-1 mRNA and RL reporters undergoing repression mediated by either miR-122 or let-7 RNA.

\section{Stress Induces HuR-dependent Relocation of CAT-1 mRNA from $P$ bodies}

mRNA reporters repressed by miRNAs were found to localize in P bodies (Liu et al. 2005; Pillai et al. 2005). We analyzed the intracellular localization of the endogenous CAT-1 mRNA and RL-cat reporters in cells grown under different conditions. In situ hybridization revealed that in nonstarved Huh7 cells, CAT-1 mRNA is concentrated in P bodies, as demonstrated by its colocalization with the P-body marker, GFP-Dcpla (Fig. 4A). P-body enrichment of the mRNA was abolished when cells were transfected with the $2^{\prime}$-O-methyl oligonucleotide complementary to miR-122 but not with control anti-miR-15 oligonucleotide (Fig. 4B), indicating that the localization was dependent on miR122. Most importantly, in Huh7 cells grown for 2 hours under amino acid deficiency, a condition that markedly increases CAT-1 protein without an effect on the mRNA level (see Fig. 1), CAT-1 mRNA was no longer detectable in $\mathrm{P}$ bodies (Fig. 4A). Starvation did not produce an appreciable decrease in the miR-122 signal in $P$ bodies (Bhattacharyya et al. 2006), arguing for an effect specific for the CAT-1 mRNA and possibly only a limited number of other mRNAs among the many regulated by miR-122 in liver cells (Krutzfeldt et al. 2005). As expected, CAT-1 mRNA did not colocalize to P bodies in HepG2 cells. However, in HepG2 cells transfected with miR-122, CAT-1 mRNA was enriched in P bodies, further supporting the idea that the repression and Pbody localization of CAT-1 mRNA are controlled by miR-122 (Bhattacharyya et al. 2006).

To find out whether the relocation of CAT-1 mRNA from $\mathrm{P}$ bodies, like the activation of its expression, also requires HuR, we studied CAT-1 distribution in Huh7 cells in which HuR protein had been knocked down by RNAi. The knockdown had no effect on the P-body 

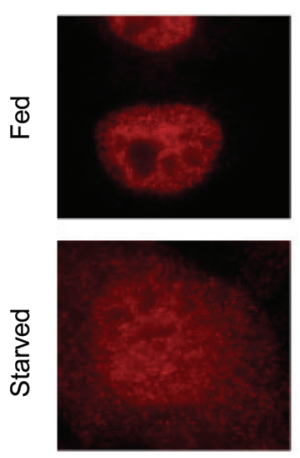

B

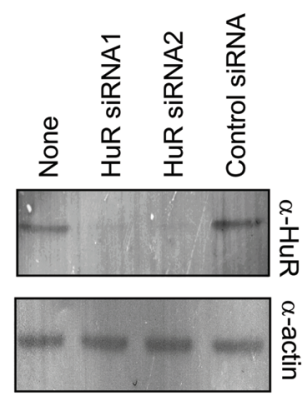

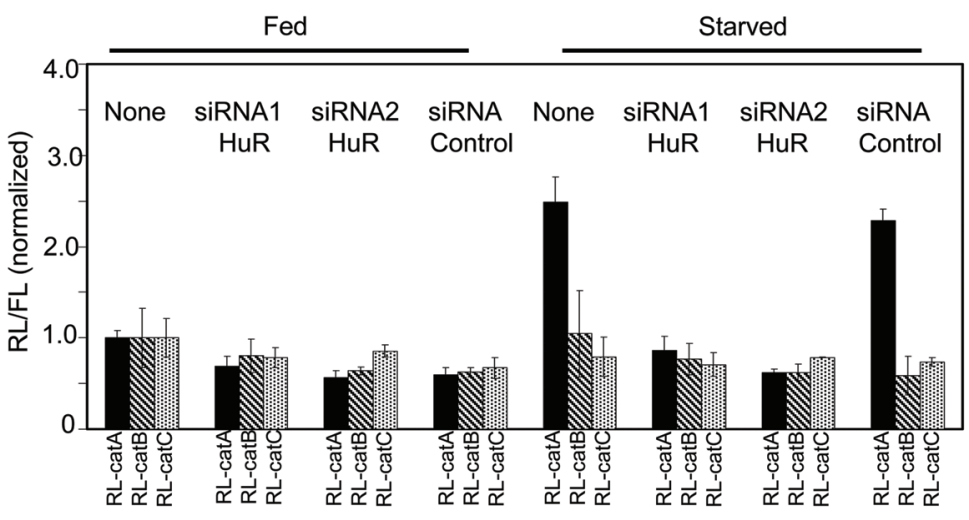

D

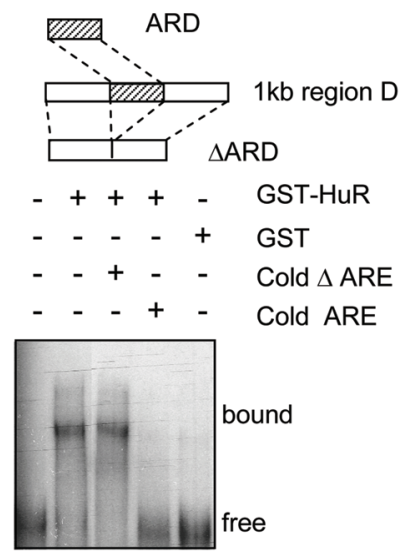

Figure 3. HuR relocates from the nucleus to the cytoplasm in stressed Huh7 cells, interacts with the CAT-1 3'UTR, and is required for the stress-induced derepression of the RL-catA reporter. (A) Starvation-induced relocation of HuR. Huh7 cells, either nonstarved (Fed) or starved for 2 hours (Starved) were fixed, and the localization of HuR (red) was determined using mouse anti-HuR monoclonal antibodies. $(B)$ Two different siRNAs effectively deplete HuR. Western blot was performed 72 hours after siRNA transfection. $(C)$ Knockdown of HuR prevents starvation-induced derepression of RL-catA. Huh7 cells were cotransfected with RL-cat reporters and indicated siRNAs; 72 hours after transfection, cells were transferred for an additional 2 hours to a medium either with or without amino acids. The values are means from three transfections $+/$ - standard deviation. (D) Recombinant GST-HuR protein interacts with the CAT-1 ARD fragment. ${ }^{32} \mathrm{P}$-labeled ARD RNA was incubated with either purified GST-HuR or GST alone in the absence or presence of indicated cold competitors, and complexes were analyzed on a native gel. (Reprinted, with permission, from Bhattacharyya et al. 2006 [C Elsevier].)

enrichment of CAT-1 mRNA in control cells. However, it prevented mobilization of the mRNA from these structures upon amino acid starvation. In cells transfected with nonspecific siRNA, the relocation did take place, similarly as in nontreated Huh7 cells (Fig. 4C).

We also determined the intracellular localization of different RL reporters repressed by either miR-122 or let-7 miRNA. The repressed reporters localized to $\mathrm{P}$ bodies, and their relocalization from these structures in response to amino acid depletion was dependent on the CAT-1 region D present downstream from the miRNAbinding sites. Reporters devoid of region $\mathrm{D}$ remained enriched in $\mathrm{P}$ bodies in both nonstarved and starved cells (Bhattacharyya et al. 2006).

In starved Huh7 cells, CAT-1 mRNA becomes extractable from cells permeabilized with digitonine, very likely as a consequence of the relocation of mRNA from $\mathrm{P}$ bodies to the cytosol (Pillai et al. 2005; Bhattacharyya et al. 2006). As shown in Figure 5A, the amount of CAT-1 mRNA present in the cytosol prepared from permeabilized Huh 7 cells increased already after 20 minutes following the shift to the amino-acid-depleted medium. Importantly, the shift of the CAT-1 mRNA to the cytosol upon stressing the cells occurred with a kinetics similar to the appearance of $\mathrm{HuR}$ in this fraction and also paralleled the accumulation of CAT-1 protein (Fig. 5). Hence, the stress-induced derepression of the CAT-1 mRNA translation appears to be a rapid event.

Together, the results demonstrate that CAT-1 mRNA and RL reporters are concentrated in $\mathrm{P}$ bodies when repressed by the miRNA but are rapidly mobilized from these structures under conditions, including cellular stress, that preclude miRNA repression. The experiments further support a role for HuR and the CAT-1 mRNA region $\mathrm{D}$ in mediating the response induced by the amino acid stress. Moreover, they indicate that such a response may be a more general phenomenon, applying to different cell types and miRNA-mRNA combinations. 
A
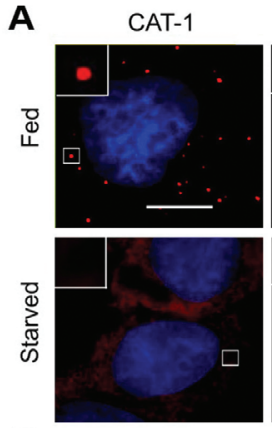

B

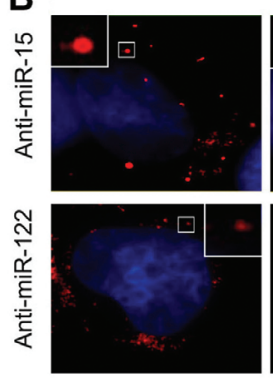

C
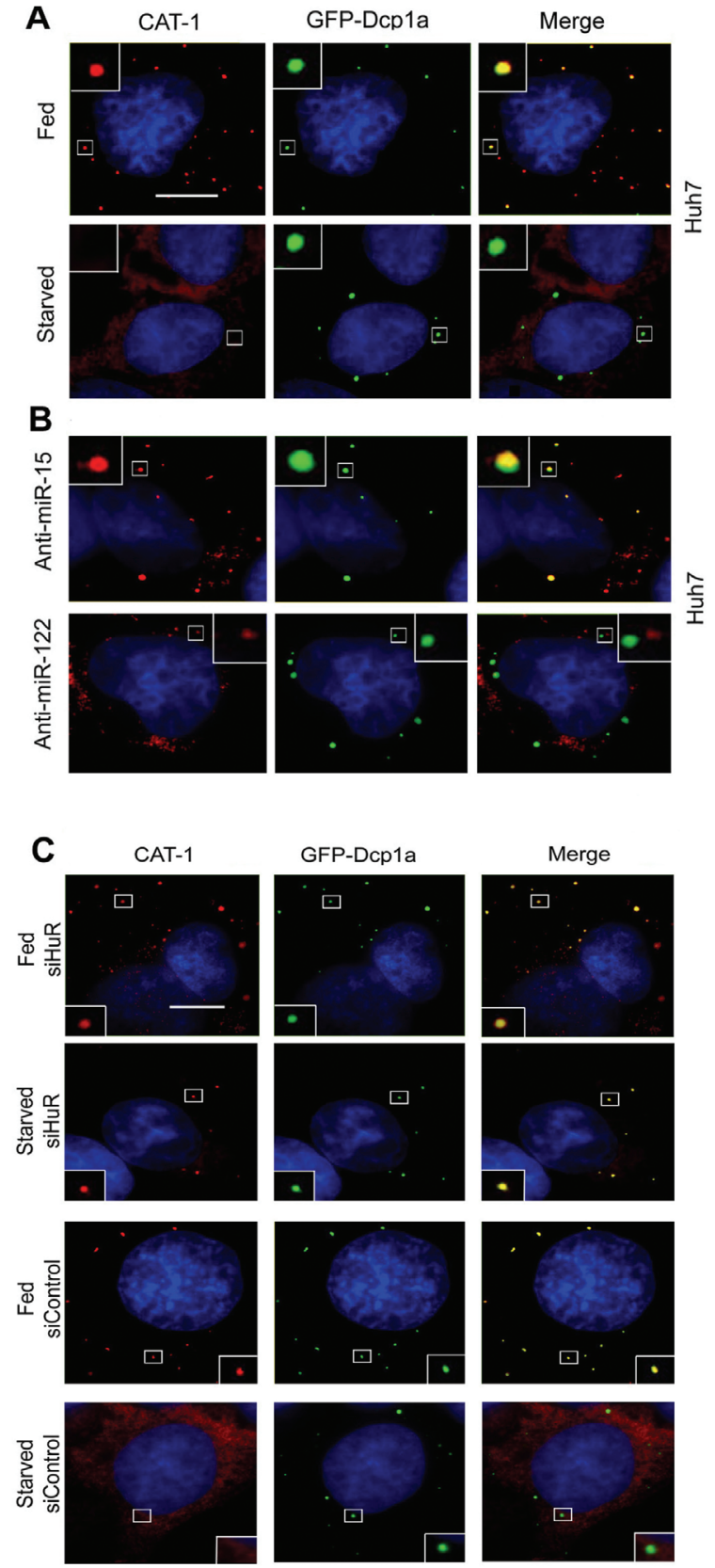

Figure 4. CAT-1 mRNA accumulation in P bodies is miR-122dependent, and its stress-induced relocation from P-bodies requires HuR. Details of cell treatment are indicated at the left of each row. P bodies were visualized by measuring GFP-Dcpla fluorescence (green), and CAT-1 by in situ hybridization with Cy3-labeled probes (red). DAPI (blue) stained the nucleus. Cells were starved for 2 hours prior to fixation. Exposure time of the red channel for starved Huh7 cells $(A)$, the anti-miR-122 row $(B)$, and starved siControl cells $(C)$ was ten times longer than that for other images. (Insets) Enlargements of indicated regions. For quantification and discussion of overlap between CAT-1 mRNA and GFP-Dcpla foci, see Bhattacharyya et al. (2006). (A,B) CAT-1 mRNA is mobilized from $\mathrm{P}$ bodies upon starvation of Huh7 cells $(A)$ or upon transfection with anti-miR-122 but not anti-miR-15 oligonucleotide $(B)$. Bar, $5 \mu \mathrm{m}$. $(C)$ HuR is required for the stress-induced mobilization of CAT-1 mRNA from $\mathrm{P}$ bodies. Huh7 cells were cotransfected with pGFP-Dcpla and either anti-HuR or control siRNA. After 48 hours, a fraction of the cells was starved for 2 hours. (Reprinted, with permission, from Bhattacharyya et al. 2006 [C Elsevier].)

\section{Stress-induced Relocation of CAT-1 mRNA from P Bodies Is Accompanied by Its Entry to Polysomes}

We found previously that repression of protein synthesis by let-7 RNA in HeLa cells is accompanied by a less effective entry of target reporters to polysomes, indicative of the translation initiation block (Pillai et al. 2005). Gradient analysis of Huh7 cell extracts indicated that amino acid starvation results in an increase in the fraction of CAT-1 mRNA associated with polysomes. In contrast, $\beta$-tubulin mRNA moved toward the top of the gradient in response to starvation, consistent with a general inhibitory effect of stress on translation (Fig. 6A,B). Treatment of Huh7 cells with anti-miR-122 but not control anti-let-7a 2'-O-methyl oligonucleotide resulted in the CAT-1 mRNA shift to polysomes similar to that induced by starvation (Fig. 6C). Of note, a fraction of the "repressed" CAT-1 mRNA sedimented faster than the polysome-associated CAT-1 mRNA present in stressed or anti-miR-122-treated cells. Possibly, this material represents P-body aggregates containing CAT1 mRNA.

Taken together, these data indicate that relocation of CAT-1 mRNA from P bodies and relief of the miRNAmediated repression is accompanied by recruitment of CAT-1 mRNA to polysomes, consistent with miR-122 inhibiting translational initiation.

\section{CONCLUSIONS}

Our work demonstrates that CAT-1 mRNA, and reporters bearing the CAT-1 3'UTR or its fragments, can be relieved from miR-122-mediated repression in Huh7 cells subjected to amino acid deprivation or the ER or oxidative stress. Observations that the response to the amino acid starvation stress can be recapitulated in other cell lines by either an ectopic supply of miR-122 or the use of chimeric reporters targeted by another miRNA argue for a general importance of this type of regulation. We also demonstrate that repressed CAT-1 and reporter mRNAs accumulate in P bodies in an miR122 -dependent process and that the derepression is accompanied by the release of the mRNAs from these structures. The demonstrated stress-induced mobilization of mRNAs from $\mathrm{P}$ bodies provides evidence that metazoan P bodies represent sites not only of mRNA turnover, but also of storage of translationally repressed mRNAs. So far, such evidence was available for baker's yeast, an organism lacking miRNA regulation (Brengues et al. 2005). Time-course experiments measuring the appearance of CAT-1 mRNA in a soluble cytosolic fraction and the formation of CAT-1 protein suggest that reactivation of mRNAs sequestered in $\mathrm{P}$ bodies is a very rapid process. Hence, it is tempting to speculate that $\mathrm{P}$ bodies may act as general storage sites for mRNAs, which need to be quickly mobilized into polysomes under specific cellular conditions.

Although miRNAs may affect gene expression in different ways (for review, see Pillai 2005; ValenciaSanchez et al. 2006), recent findings indicated that let-7 RNA and some model miRNAs in mammals inhibit 
A
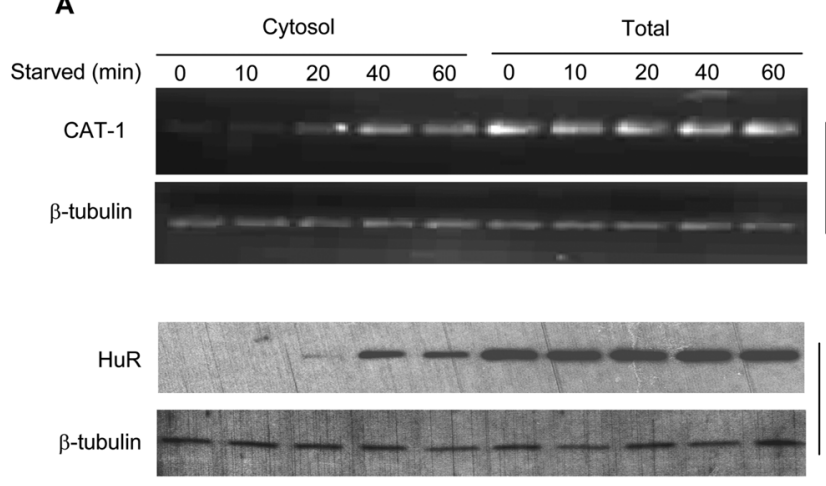

B

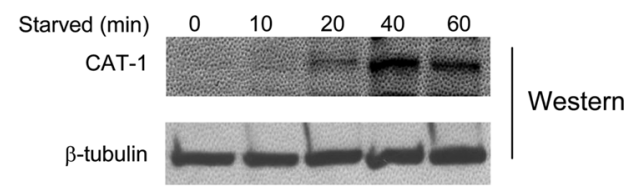

translation of reporter mRNAs at the initiation step (Humphreys et al. 2005; Pillai et al. 2005) and that repressed mRNAs localize to $\mathrm{P}$ bodies for either storage or degradation (Liu et al. 2005; Pillai 2005; Pillai et al. 2005; Valencia-Sanchez et al. 2006). The data presented in this work indicate that this scenario also applies to the miRNA-mediated regulation of an endogenous mRNA. The stress- or anti-miR-122-induced relocalization of CAT-1 mRNA from P bodies was accompanied by its increased association with polysomes, consistent with the miRNA inhibition acting at the initiation step of transla-
Western

RT-PCR

Figure 5. The stress-induced relocation of HuR protein and CAT-1 mRNA to the cytosol and the accumulation of CAT-1 protein are rapid events. (A) Accumulation of CAT-1 mRNA and HuR protein in the cytosol upon stressing Huh7 cells occurs with a similar kinetics. Huh7 cells were starved for amino acids for increasing time, and levels of CAT-1 mRNA (upper panels) and HuR protein (western; lower panels) were measured in the cytosolic and total extracts of starved cells. Cytosolic extracts were prepared by permeabilizing cells with digitonine (Bhattacharyya et al. 2006). (B) Kinetics of the CAT-1 protein accumulation in Huh7 cells subjected to amino acid deprivation stress, determined by western.
A

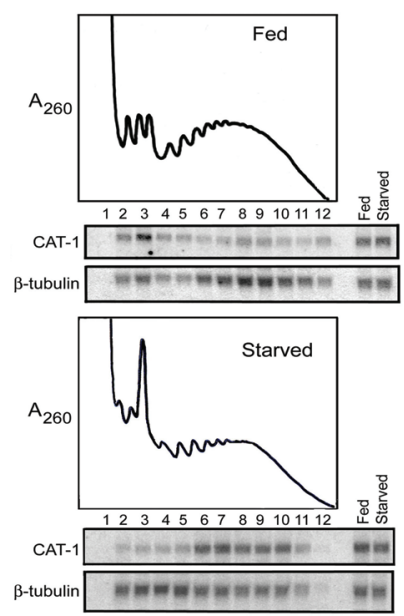

B
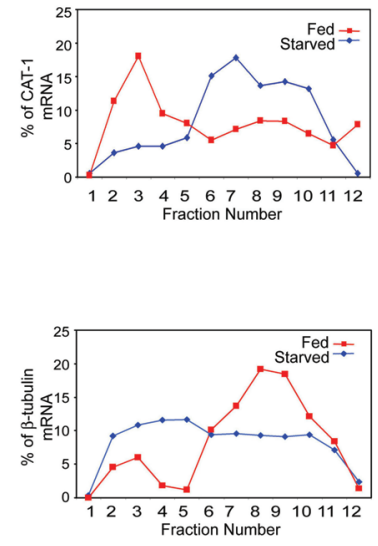

C
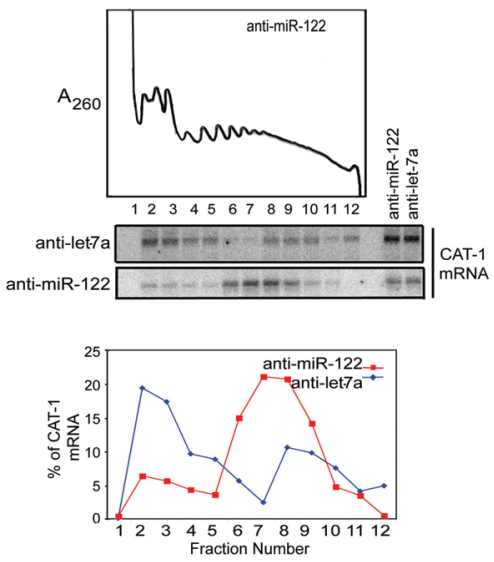

Figure 6. Stress- or anti-miR-122-induced relocation of CAT-1 mRNA from P bodies is accompanied by its recruitment to polysomes. (A) Distribution of CAT-1 mRNA in extracts from cells fed with amino acids (upper panels) or starved for amino acids (lower panels). RNA extracted from individual fractions was analyzed by northern blots with probes specific for CAT-1 and $\beta$-tubulin mRNAs. Two lanes at the right represent input RNA isolated from fed and starved cells. (B) Quantification of distribution of mRNAs analyzed in panel $A$, expressed as a percentage of total radioactivity present in each lane. $(C)$ Distribution of CAT-1 mRNA in extracts from cells treated with either anti-let-7a or anti-miR-122 (middle panels). $\mathrm{A}_{260}$ profile of the anti-let-7a extract was similar to that shown at the top, and distribution of $\beta$-tubulin mRNA in both gradients was similar to that shown in panel $A$, fed cells (data not shown). Quantification of CAT-1 mRNA is at the bottom. (Reprinted, with permission, from Bhattacharyya et al. 2006 [C Elsevier].) 
Moreover, a recombinant HuR interacted with the CAT13 'UTR fragment implicated in mediating the stimulatory effect of stress (see Fig. 3D), whereas the endogenous HuR associated with all reporter mRNAs undergoing derepression but not with their inactive variants bearing mutations in the predicted HuR-binding sites (Bhattacharyya et al. 2006). HuR is a ubiquitously expressed member of the ELAV family of proteins, which also comprises three neuronal proteins. In response to different types of cellular stress, HuR is mobilized from the nucleus to the cytosol, where it may modulate translation and/or stability of different mRNAs (for review, see Brennan and Steitz 2001; Katsanou et al. 2005; Lal et al. 2005). Our data suggest that at least some of the known effects of HuR, both translational and stability-related, may be due to the interference of $\mathrm{HuR}$ with the function of miRNAs, which would result in enhanced translation or stability of mRNA. HuR shuttles between the nucleus and cytoplasm, and HuR has been suggested to bind some AREcontaining mRNAs in the nucleus and chaperone them to the cytoplasm (Gallouzi and Steitz 2001; Lal et al. 2005). The in situ experiments demonstrating CAT-1 mRNA and RL reporter localization in $\mathrm{P}$ bodies in unstressed hepatoma and HeLa cells make it very unlikely that redistribution of mRNA between the nucleus and the cytoplasm contributes to the effects described in our work.

The demonstration that mRNAs repressed by miRNAs can depart $\mathrm{P}$ bodies to return to active translation indicates that $\mathrm{P}$ bodies are dynamic structures, exchanging their content rapidly with that of the cytosol (Andrei et al. 2005; Brengues et al. 2005). It is possible that HuR, following its relocation to the cytoplasm in stressed cells, shifts the P-body-to-cytosol equilibrium of repressed mRNAs by binding to AREs in the $3^{\prime} U T R$. Whether this is accompanied by the dissociation of miRNPs from the mRNA or just prevents miRNPs from acting as effectors in the repression remains to be established (Fig. 7). It will also be interesting to study other details of HuR involvement in relieving the miRNP repression. The findings that several identified protein ligands of HuR are protein phosphatase PP2A inhibitors (Brennan et al. 2000) and that HuR can undergo methylation (Li et al. 2002) or synergize with other RNA-binding proteins (Katsanou et al. 2005) indicate that HuR is a part of an elaborated network involved in posttranscriptional regulation of gene expression.

Other examples of the reversible action of miRNAs have recently been identified in neuronal cells. In neurons, many mRNAs are transported along the dendrites as repressed mRNPs to become translated at the final destination, dendritic spines, upon synaptic activation. Such local translation is important for spine development, learning, and memory (Sutton and Schuman 2005). miRNA miR-134 is implicated in translational regulation of Limk1, a protein kinase important for spine development, in cultured rat neurons. Limk1 mRNA appears to be relieved from the miR-134-mediated repression in dendritic spines in response to extracellular stimuli, in a process involving mTOR (mammalian target of rapamycin) (Schratt et al. 2006). In Drosophila, stimulation of olfactory neurons, which leads to long-term memory formation, is associated with proteolysis of Armitage, a protein essential for the assembly of the RNA-induced silencing complex (RISC)/miRNP complexes. As the result of Armitage degradation, mRNAs that are normally repressed by miRNAs, including the one encoding calcium/calmodulin-dependent protein kinase II (CamKII), become

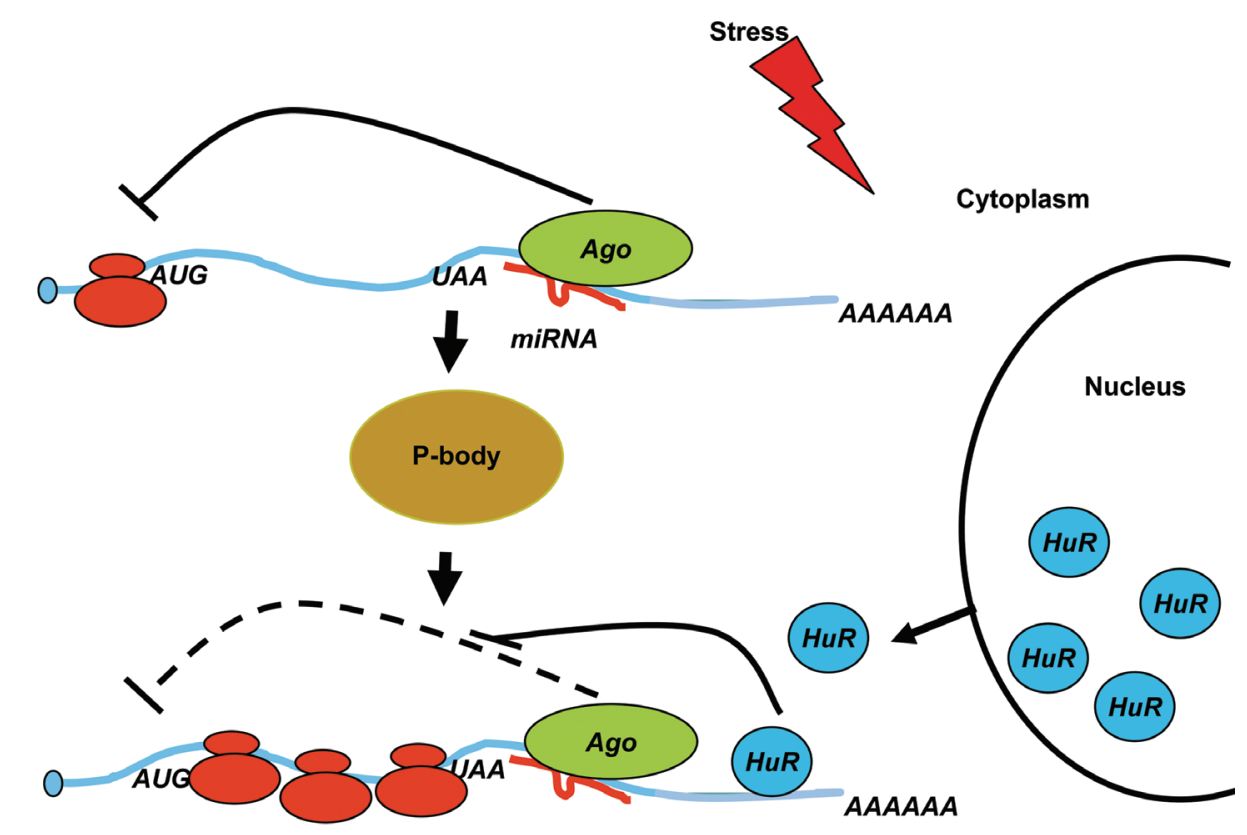

Figure 7. Model of the stress- and HuR-mediated relief of CAT-1 mRNA repression by miR-122. 
effectively translated in the synapse (Ashraf et al. 2006). The regulation in Drosophila differs from the examples studied in mammalian cells, in that the Armitage depletion most probably indiscriminately prevents the formation of repressed mRNPs, rather than causing reactivation of specific mRNAs controlled by miRNAs.

In addition to $\mathrm{HuR}$, three other ELAV proteins, $\mathrm{HuA}, \mathrm{HuB}$, and $\mathrm{HuD}$, are expressed in neurons, and a role of $\mathrm{HuD}$ in stability and translation of some neuronal mRNAs has already been documented (PerroneBizzozero and Bolognani 2002). It will be interesting to find out whether, similarly to HuR in hepatoma cells, the other ELAV proteins modulate miRNAmediated regulation in neurons. Likewise, it will be important to determine whether other classes of RNAbinding proteins interacting with the $3^{\prime} \mathrm{UTR}$ will act as modifiers altering the potential of miRNAs to repress gene expression.

\section{ACKNOWLEDGMENTS}

We thank C. Clayton, T. Hobman, Y. Nagamine, C. Ender, R. Pillai, C. Artus, and E. Bertrand for providing plasmids and/or antibodies. S.N.B. is a recipient of a longterm HFSP fellowship. The Friedrich Miescher Institute is supported by the Novartis Research Foundation.

\section{REFERENCES}

Andrei M.A., Ingelfinger D., Heintzmann R., Achsel T., Rivera-Pomar R., and Luhrmann R. 2005. A role for eIF4E and eIF4E-transporter in targeting mRNPs to mammalian processing bodies. RNA 11: 717 .

Ambros V. 2004. The functions of animal microRNAs. Nature 431: 350 .

Ashraf S.I., McLoon A.L., Sclarsic S.M., and Kunes S. 2006. Synaptic protein synthesis associated with memory is regulated by the RISC pathway in Drosophila. Cell 124: 191.

Bartel D.P. 2004. MicroRNAs: Genomics, biogenesis, mechanism, and function. Cell 116: 281.

Bhattacharyya S.N., Habermacher R., Martine U., Closs E.I., and Filipowicz W. 2006. Relief of microRNA-mediated translational repression in human cells subjected to stress. Cell 125: 1111.

Brengues M., Teixeira D., and Parker R. 2005. Movement of eukaryotic mRNAs between polysomes and cytoplasmic processing bodies. Science 310: 486.

Brennan C.M. and Steitz J.A. 2001. HuR and mRNA stability. Cell. Mol. Life Sci. 58: 266.

Brennan C.M., Gallouzi I.E., and Steitz J.A. 2000. Protein ligands to HuR modulate its interaction with target mRNAs in vivo. J. Cell Biol. 151: 11.

Chang J., Nicolas E., Marks D., Sander C., Lerro A., Buendia M.A., Xu C., Mason W.S., Moloshok T., Bort R., et al. 2004. miR-122, a mammalian liver-specific microRNA, is processed from hcr mRNA and may downregulate the high affinity cationic amino acid transporter CAT-1. RNA Biol. 1: 106.

Filipowicz W. 2005. RNAi: The nuts and bolts of the RISC machine. Cell 122: 17.

Gallouzi I.E. and Steitz J.A. 2001. Delineation of mRNA export pathways by the use of cell-permeable peptides. Science 294: 1895.

Hatzoglou M., Fernandez J., Yaman I., and Closs E. 2004. Regulation of cationic amino acid transport: the story of the CAT-1 transporter. Annu. Rev. Nutr. 24: 377.
Humphreys D.T., Westman B.J., Martin D.I., and Preiss T. 2005. MicroRNAs control translation initiation by inhibiting eukaryotic initiation factor 4E/cap and poly(A) tail function. Proc. Natl. Acad. Sci. 102: 16961.

Jakymiw A., Lian S., Eystathioy T., Li S., Satoh M., Hamel J.C., Fritzler M.J., and Chan E.K. 2005. Disruption of GW bodies impairs mammalian RNA interference. Nat. Cell Biol. 7: 1267.

Katsanou V., Papadaki O., Milatos S., Blackshear P.J., Anderson P., Kollias G., and Kontoyiannis D.L. 2005. HuR as a negative posttranscriptional modulator in inflammation. Mol. Cell 16: 777 .

Krutzfeldt J., Rajewsky N., Braich R., Rajeev K.G., Tuschl T., Manoharan M., and Stoffel M. 2005. Silencing of microRNAs in vivo with "antagomirs." Nature 438: 685.

Lal A., Kawai T., Yang X., Mazan-Mamczarz K., and Gorospe M. 2005. Antiapoptotic function of RNA-binding protein HuR effected through prothymosin alpha. EMBO J. 24: 1852.

Li H., Park S., Kilburn B., Jelinek M.A., Henschen-Edman A., Aswad D., Stallcup M.R., and Laird-Offringa I.A. 2002. Lipopolysaccharide-induced methylation of $\mathrm{HuR}$, an mRNA-stabilizing protein, by CARM1. J. Biol. Chem. 277: 44623.

Liu J., Valencia-Sanchez M.A., Hannon G.J., and Parker R. 2005. MicroRNA-dependent localization of targeted mRNAs to mammalian P-bodies. Nat. Cell Biol. 7: 719.

Liu J., Carmell M.A., Rivas F.V., Marsden C.G., Thomson J.M., Song J.J., Hammond S.M., Joshua-Tor L., and Hannon G.J. 2004. Argonaute2 is the catalytic engine of mammalian RNAi. Science 305: 1437.

Meister G., Landthaler M., Patkaniowska A., Dorsett Y., Teng G., and Tuschl T. 2004. Human Argonaute2 mediates RNA cleavage targeted by miRNAs and siRNAs. Mol. Cell 15: 185.

Olsen P.H. and Ambros V. 1999. The lin-4 regulatory RNA controls developmental timing in Caenorhabditis elegans by blocking LIN-14 protein synthesis after the initiation of translation. Dev. Biol. 216: 671.

Perrone-Bizzozero N. and Bolognani F. 2002. Role of HuD and other RNA-binding proteins in neural development and plasticity. J. Neurosci. Res. 68: 121.

Petersen C.P., Bordeleau M.E., Pelletier J., and Sharp P.A. 2006. Short RNAs repress translation after initiation in mammalian cells. Mol. Cell 21: 533.

Pillai R.S. 2005. MicroRNA function: Multiple mechanisms for a tiny RNA? RNA 11: 1753.

Pillai R.S., Artus C.G., and Filipowicz W. 2004. Tethering of human Ago proteins to mRNA mimics the miRNA-mediated repression of protein synthesis. RNA 10: 1518.

Pillai R.S., Bhattacharyya S.N., Artus C.G., Zoller T., Cougot N., Basyuk E., Bertrand E., and Filipowicz W. 2005. Inhibition of translational initiation by let-7 miRNA in human cells. Science 309: 1573.

Schratt G.M., Tuebing F., Nigh E.A., Kane C.G., Sabatini M.E., Kiebler M., and Greenberg M.E. 2006. A brainspecific miRNA regulates dendritic spine development. Nature 439: 283.

Sutton M.A. and Schuman E.M. 2005. Local translational control in dendrites and its role in long-term synaptic plasticity. J. Neurobiol. 64: 116.

Tomari Y. and Zamore P.D. 2005. Perspective: Machines for RNAi. Genes Dev. 19: 517.

Valencia-Sanchez M.A., Liu J., Hannon G.J., and Parker R. 2006 Control of translation and mRNA degradation by miRNAs and siRNAs. Genes Dev. 20: 515.

Wienholds E. and Plasterk R.H. 2005. MiRNA function in animal development. FEBS Lett. 579: 5911.

Yaman I., Fernandez J., Sarkar B., Schneider R.J., Snider M.D., Nagy L.E., and Hatzoglou M. 2002. Nutritional control of mRNA stability is mediated by a conserved AU-rich element that binds the cytoplasmic shuttling protein HuR. $J$. Biol. Chem. 277: 41539. 


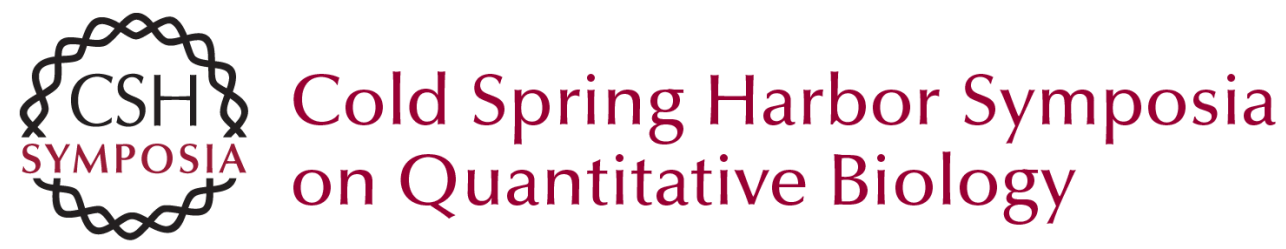

\section{Stress-induced Reversal of MicroRNA Repression and mRNA P-body Localization in Human Cells}

S.N. BHATTACHARYYA, R. HABERMACHER, U. MARTINE, et al.

Cold Spring Harb Symp Quant Biol 2006 71: 513-521

Access the most recent version at doi:10.1101/sqb.2006.71.038

References This article cites 33 articles, 12 of which can be accessed free at: http://symposium.cshlp.org/content/71/513.full.html\#ref-list-1

License

Email Alerting

Receive free email alerts when new articles cite this article - sign up in Service the box at the top right corner of the article or click here. 